The American Journal of Sports Medicine, Vol. 7, No. 4

(C) 1979 American Orthopaedic Society for Sports Medicine

\title{
The saphenous nerve: its course and importance in medial arthrotomy*
}

\author{
LETHA Y. HUNTER,† M.D., DEAN S. LOUIS, M.D., JAMES R. RICCIARDI, $\ddagger$ M.D., AND \\ GERALD A. O'CONNOR, M.D., Ann Arbor, Michigan \\ From the Department of Surgery, Section of Orthopaedic Surgery, University of Michigan, \\ Ann Arbor, Michigan
}

\begin{abstract}
Both lower extremities of 10 cadavers (20 specimens) were dissected to delineate the course of the saphenous nerve and its two major divisions (sartorial and infrapatellar branches). The course of the saphenous nerve followed the standard text description, except at the point in the sartorius muscle where the infrapatellar branch exited to become a subcutaneous structure. The location of this branch varied slightly in each cadaver but was the same for both lower extremities in the same cadaver. The location of the sartorial nerve and its relationship to the tendons of the pes anserinus was consistent in all 20 specimens. Since $69 \%$ of a group of 75 patients found altered sensation significant after routine sectioning of the infrapatellar nerve following medial meniscectomy, a group of surgeons at the University of Michigan is now protecting the infrapatellar branch of the saphenous nerve at operation. Early results on a small number of patients indicate that no alteration in sensation occurs if the nerve is carefully retracted.
\end{abstract}

he saphenous nerve is the sensory branch of the posterior division of the femoral nerve. It is the longest cutaneous branch of the femoral nerve and is considered its major branch. It supplies sensation to the anteromedial aspect of the leg primarily through two major divisions: the sartorial and infrapatellar nerves (Fig. 1). The proximity of these two nerves to the

\footnotetext{
* Presented at the Fourth Annual Meeting of the American Orthopaedic Society for Sports Medicine, Lake Placid, New York, June 30 to July 4,1978 .

$\dagger$ Address correspondence to: Department of Surgery, Section of Orthopaedic Surgery, University of Michigan, Ann Arbor, Michigan 48109 .

$\ddagger$ Doctor Ricciardi is currently at Maryville Orthopaedic Clinic,

P.C., Maryville, Tennessee.
}

knee makes knowledge of their location important in medial arthrotomies.

\section{ANATOMY}

In the proximal thigh the saphenous nerve lies lateral to the femoral artery (Fig. 2A). It then disappears into the adductor canal in the adductor magnus muscle where it crosses medially over the femoral artery (Fig. 2B). It exits from the canal with the saphenous branch of the inferior geniculate artery and divides almost immediately into its infrapatellar and sartorial branches (Fig. 2C).

The posterior or infrapatellar branch of the saphenous nerve pierces the sartorius muscle and then takes an oblique subcutaneous course to a subpatellar position at the medial border of the patella (Fig. 2D).

At this point the nerve terminates in multiple small branches that comprise the major portion of the infrapatellar nerve plexus. This plexus supplies sensation to the inferior patellar area.

The anterior or sartorial branch of the saphenous nerve becomes subcutaneous by exiting between the sartorius and gracilis tendons. After giving off a major branch to supply sensation to the skin over the pes anserinus (Fig. 2D), it takes a fairly vertical course down the leg to lie anterior to the medial malleolus and arborizes at approximately the level of the metatarsophalangeal joint of the great toe. Throughout its course it gives off many small cutaneous branches.

\section{DISSECTION STUDIES}

We dissected both lower extremities of 10 cadavers, a total of 20 specimens. We found in all specimens that the course of the saphenous nerve was consistent with the description in standard texts of anatomy ${ }^{1-3}$ until the point where the infrapatellar branch exited to become a subcutaneous structure. This point varied slightly in each cadaver but was the same for both 


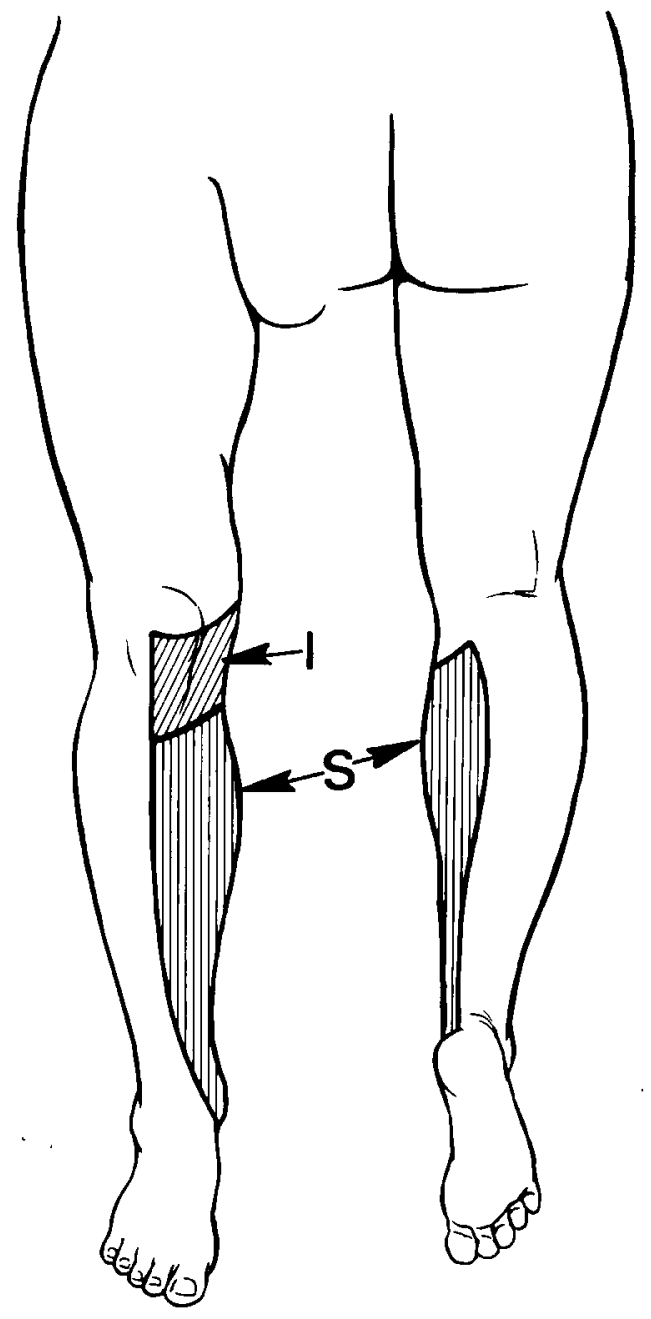

Fig. 1. Sensory distribution of the saphenous nerve (patterned after Cunningham's Textbook of Anatomy). ${ }^{8} \mathrm{I}$, infrapatellar distribution; $S$, sartorial distribution.

extremities of the same cadaver. In one of the cadavers, the infrapatellar nerve exited so distally in the sartorius muscle that it actually came through the tendinous portion of the muscle; thus it had a more transverse course to reach a subpatellar position at the medial border than the branches in the other specimens. The location of the sartorial nerve and its relationship to the tendons of the pes anserinus was consistent in all 20 specimens examined.

\section{COMMENT}

Protection of the sartorial nerve during medial arthrotomy is well recognized. Transecting this nerve will result in anesthesia throughout the anteromedial aspect of the leg. When performing a pes-plasty, one should be careful to mobilize the pes anserinus by incising only the deep fascia along the lower border of the semitendinosus. The sartorial nerve having exited from the deep structures between the sartorius and gracilis muscles will be superficial to the plane of dissection and thus be protected in the subcutaneous tissue (Fig. 3).

The need for protection of the infrapatellar branch of the saphenous nerve is not as well established. Abbott and Carpenter ${ }^{4}$ in their classic article on surgical approaches to the knee joint published in 1945 stated: "Division of the infrapatellar branch of the saphenous nerve at the time of an operation for removal of the internal semilunar cartilage may cause a painful neuroma with symptoms which are similar to those for which the cartilage was removed." They suggested that the surgeon should protect the nerve when performing arthrotomies. More recently Larson ${ }^{5}$ expressed the opinion of others when he wrote: "The infrapatellar branch of the saphenous nerve ... is routinely sectioned with the medial incision used. Its loss is of no clinical importance."

The only reported statistical study of the significance of transection of the infrapatellar nerve is by Johnson et al., ${ }^{6}$ and this is included in their paper on late results of meniscectomy. They found that of the 76 patients who had medial incisions, 35 had no decreased sensation; 18 had decreased sensation but felt it was of no concern; 16 reported nonincapacitating irritation when kneeling; and 7 patients claimed they could not kneel on the knee because of the paresthesia over the area of the tibial tubercle. Or to state these results differently, 41 of 76 patients had altered sensation, but only 23 of 76 patients considered this alteration significant. No mention was made as to whether or not the infrapatellar nerve was retracted or sectioned in their patients.

At the University of Michigan, we routinely section the infrapatellar nerve when making a medial parapatellar incision. To determine the significance of sacrificing this structure, 75 patients, undergoing medial arthrotomy in which the standard medial parapatellar incision had been performed, were surveyed with the following results: 8 had no altered sensation; 15 had altered sensation but found it of no concern; 47 reported nonincapacitating irritation especially when kneeling; and 5 patients claimed they could not kneel because of paresthesia. That is, 67 of 75 had altered sensation following their arthrotomy, and 57 of 75 found this altered sensation significant. Sixteen percent of the patients said acceptance of the area of altered sensation had improved with time. Only $10 \%$ felt that the total area of altered sensation had significantly decreased during their first postoperative year. Age made no difference in accepting the change in sensation in the infrapatellar area.

The important statistic, we believe, is that $69 \%$ of the patients felt their altered sensation was significant to them. It certainly would be beneficial if we could decrease this morbidity even though in most cases it was only an annoying and not an incapacitating morbidity.

Therefore, we are presently attempting to preserve the infrapatellar nerve during medial arthrotomies. We have found it difficult to visualize and retract the nerve when using the standard medial parapatellar skin incision or the posterior medial skin incision because both of these cross the nerve at the right angles (Fig. 4).

The Bosworth incision ${ }^{7}$ extends obliquely from the adductor tubercle of the femur to the medial border of the tibial tubercle (Fig. 5). Through this skin incision one can make both a 

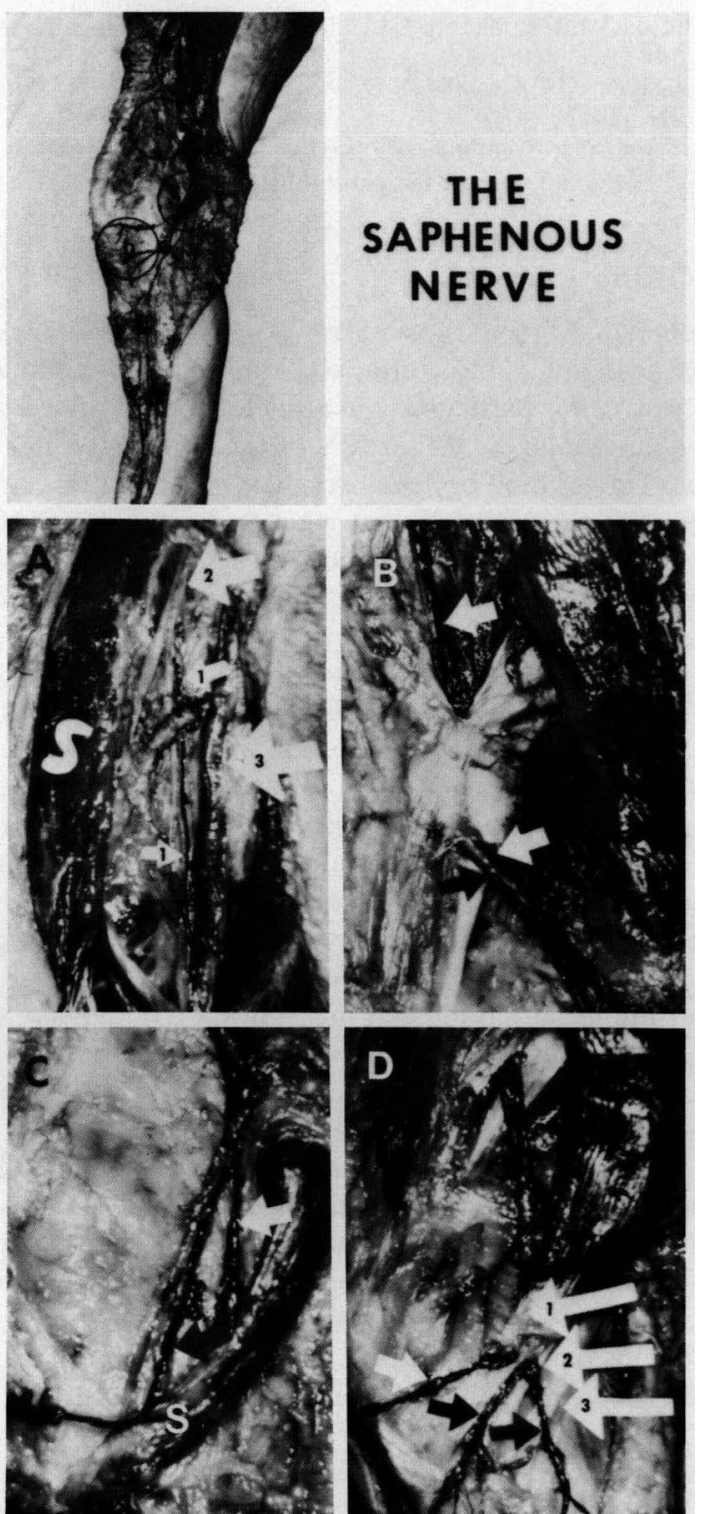

Fig. 2. An overview of the course of the saphenous nerve. The nerve is traced with black ink. The areas circled correspond to the lettered enlarged views shown. A. The saphenous nerve is seen originating from the femoral nerve below the inguinal ligament. Note the femoral nerve (2), the saphenous nerve (1), the femoral artery (3), and the sartorius muscle (S) which has been retracted laterally. B. The saphenous nerve (white arrow) is seen entering the adductor canal and then exiting with the saphenous branch of the inferior geniculate artery (black arrow). C. After exiting from the adductor canal, the saphenous nerve divides into an anterior or sartorial division (black arrow) and a posterior or infrapatellar division (white arrow). $S$, sartorius muscle. $D$. The infrapatellar nerve (small white arrow) pierces the sartorius muscle or tendon and then travels subcutaneously to terminate infrapatellarly, supplying sensation to the skin in this area. The sartorial nerve (black arrow) becomes subcutaneous by exiting between the sartorius and gracilis tendons. It immediately gives off a major branch to

Vol. 7, No. 4, 1979

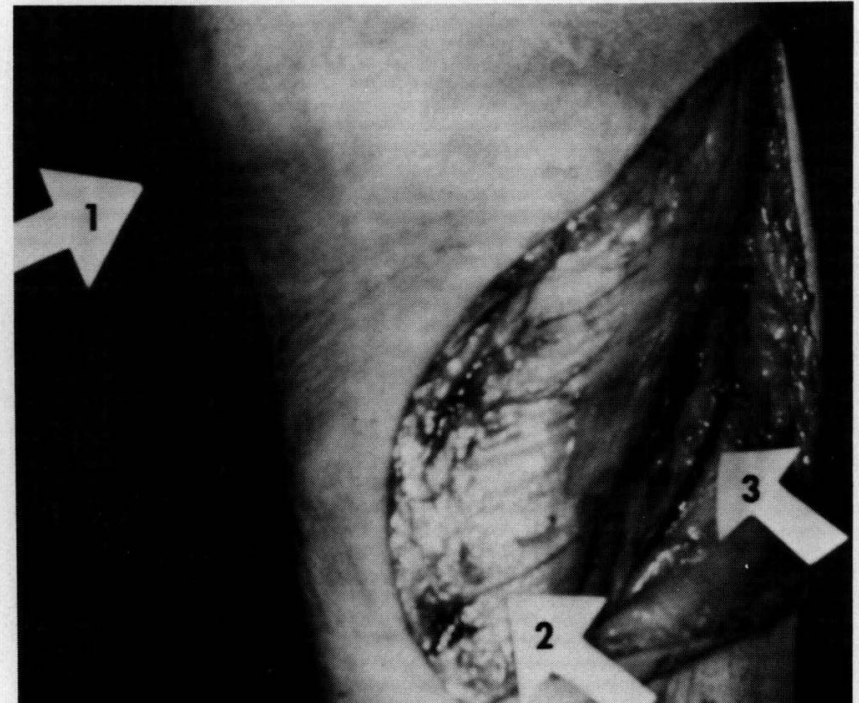

Fig. 3. Anteromedial view of the knee demonstrating the relationship of the inferior border of the pes anserinus (2) to the sartorial nerve (3). For orientation, the inferior border of the patella is labeled (I).

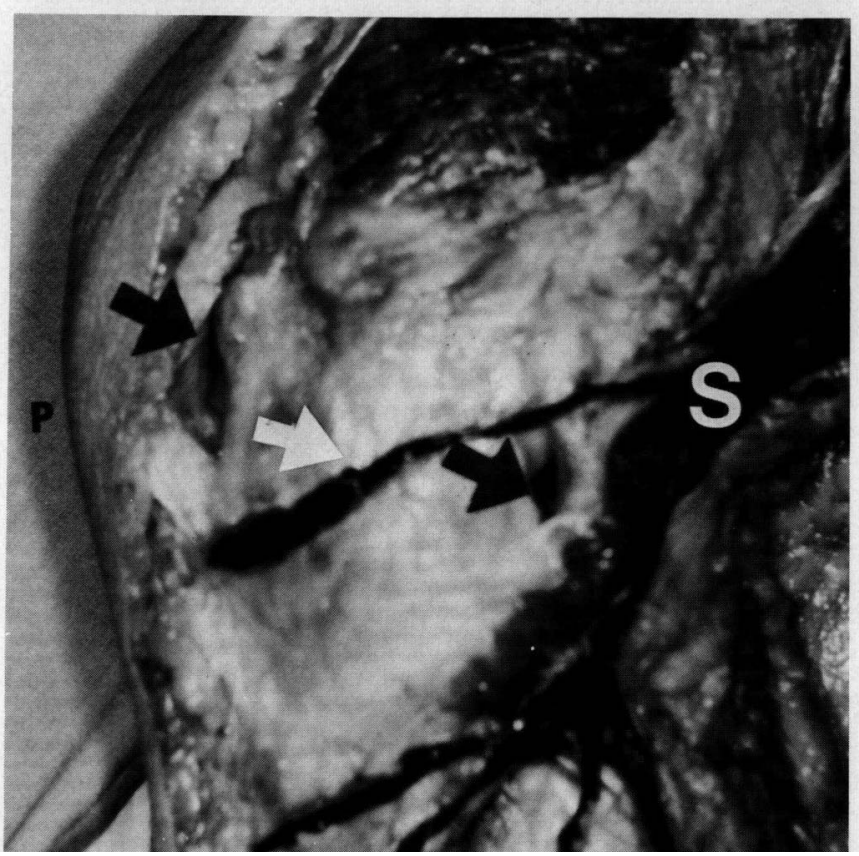

Fig. 4. Medial side of the knee illustrating the relationship of the infrapatellar nerve (white arrow) to anterior and posterior capsular incisions (black arrows). $S$, sartorius muscle; $P$, inferior border of the patella.

supply sensation to the skin over the pes anserinus (black arrow on the left) and then continues anteromedially down the leg, giving off many small sensory branches throughout its course The three large areas point to the tendons of the pes anserinus: (1) sartorius, (2) gracilis, (3) semitendinosus.

The Saphenous Nerve 


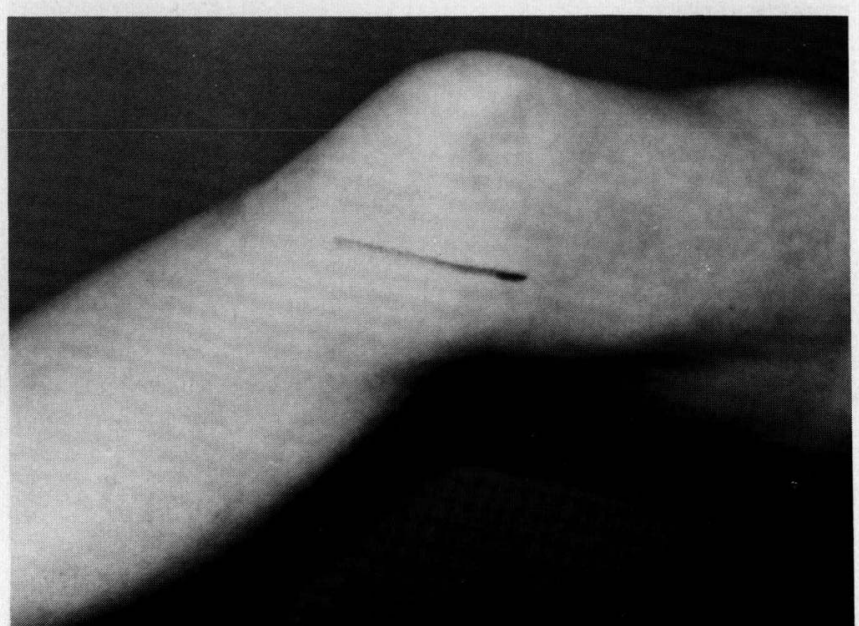

Fig. 5. Medial side of knee depicting Bosworth incision extending from the femoral adductor tubercle to the medial border of the tibial tubercle.

parapatellar capsular incision as well as a posterior medial capsular incision. The skin incision can also be extended down the tibial shaft for greater exposure.

The course of the infrapatellar nerve is similar to the direction of this incision, making the nerve visible throughout the length of the incision. This facilitates identification and retraction of the nerve.

Only after surveying the patients in whom we are now protecting the infrapatellar nerve will we be able to answer the question of how much morbidity, if any, is associated with gently retracting the nerve during arthrotomy. Early results on a small number of patients demonstrate no alteration in sensation if the nerve is carefully retracted.

We are all aware that sensation in the upper extremity is extremely important, and we take great efforts to protect upper extremity cutaneous nerves. Perhaps a greater effort should be made to protect sensation in the lower extremity especially in the "kneeling sensitive" area of the knee. We are hoping to find less morbidity associated with retracting the infrapatellar nerve than $69 \%$ morbidity associated with cutting it

\section{REFERENCES}

1. Anson BJ: An Atlas of Human Anatomy. Second edition. Philadelphia, WB Saunders, 1963, pp 472-473

2. Grant JCB: Grant's Atlas of Anatomy. Sixth edition. Baltimore, Williams \& Wilkins Co, 1972, pp 248-263

3. Woodburne RT: Essentials of Human Anatomy. Fourth edition. London, Oxford University Press, 1969, p 528

4. Abbott LC, Carpenter WF: Surgical approaches to the knee joint. J Bone Joint Surg 27A: 277-310, 1945

5. Larson R: In Injuries of the Ligaments of the Knee. Edited by CA Rockwood and DP Green, Philadelphia, JB Lippincott, 1975, p 1256
6. Johnson RJ, Kettlekamp DB, Clark W, et al: Factors affecting late results after meniscectomy. J Bone Joint Surg 56A: 719-729, 1974

7. Crenshaw AH: Campbell's Operative Orthopaedics. St. Louis, CV Mosby, 1971

8. Romanes GJ: Cunningham's Textbook of Anatomy. Eleventh edition. London, Oxford Medical Publications, 1972, p 760

\section{EDITORIAL COMMENT}

Dr. Robert L. Larson: Most of us who do meniscectomies have felt that sectioning of the infrapatellar branch of the saphenous nerve was of no particular consequence. Dr. Hunter and coworkers have suggested that $69 \%$ of the patients do not agree with us. The terminology she uses that $69 \%$ found this "altered sensation significant" was somewhat unspecific. One can look at hers in another light, in which only $6.6 \%$ had paresthesias interfering with function and $93.4 \%$ had no incapacitating problems.

My advice about the infrapatellar branch of the saphenous nerve problem has always been either to cut it or gently protect it so that a neuroma does not develop. To this, in view of our recent practiced climate, I would add the advice to tell the patients that they may have numbness or paresthesias over the tibial tubercle area which would either gradually return to normal sensation or they would tend to get used to it. Dr. Hunter's figures show that only about $10 \%$ after 1 year will have a change of mind about the significance.

With the usual paramedial incision, Dr. Hunter has shown that anatomically, if the excision is not extended past $1 \mathrm{~cm}$ below the joint line, the infrapatellar branch will be safe. One should probably make this effort to avoid its injury.

I do not use the Bosworth incision because of the length necessary for a meniscectomy and, if I do desire to extend my incision, I would prefer to create a posterior skin flap with a more vertical-anterior orientation. This allows me to work on the patella if necessary. If a distal extension is necessary for ligamentous reconstruction, the patellar branch is usually sacrificed because, even though it can be identified and protected, it usually gets torn in the surgical procedure.

The sartorial branch of the saphenous nerve, on the other hand, can be identified and protected since it supplies a large area of sensation and may be entrapped with various tendon transfers that are done.

Dr. Hunter and her co-workers have presented a refreshingly simple problem and have investigated the area of the problem anatomically, assayed the clinical ramifications, and suggested a method of reducing or eliminating the problem in simple meniscectomies. They have also recognized that the study is not complete until the results of the change in technique are evaluated or if the longer incisional scar created by the Bosworth procedure produces dissatisfaction in a certain percentage of patients, for one reason or another.

Authors' Reply: Thank you, Dr. Larson, for your comments. 